\section{Sobrevida de pessoas com hantavirose diagnosticadas no Estado do Paraná, Brasil}

\author{
Survival of persons with hantavirus infection \\ diagnosed in Paraná State, Brazil
}

\section{Supervivencia de personas con hantavirus \\ diagnosticadas en el estado de Paraná, Brasil}

COMUNICAÇÃO BREVE

BRIEF COMMUNICATION
Daniele Akemi Arita 1

Silvia Emiko Shimakura 1

doi: 10.1590/0102-311X00105518

\section{Correspondência}

D. A. Arita

Rua Sete de Setembro 514, apto. 504, Pinhais, PR 83323-270,

Brasil.

danielearita2002@yahoo.com.br tras doenças, seu prognóstico poderia ser melhorado por meio do conhecimento de fatores que pudessem contribuir para seu desfecho. O estudo objetivou identificar os fatores associados ao tempo de sobrevivência em pessoas com hantavirose. Trata-se de um estudo com casos confirmados de hantavirose de janeiro/1992 a junho/2016 do Estado do Paraná, Brasil. Cada caso apresentou dois desfechos competitivos possíveis: óbito ou cura. Para a análise de sobrevivência foi utilizado um modelo de riscos competitivos e as probabilidades dos desfechos estimadas pelo estimador de Aalen-Johansen. A medida de associação foi a razão de riscos (RzRiscos), com respectivos intervalos de $95 \%$ de confiança (IC95\%). Dos 280 indivíduos com hantavirose, 107 (38,21\%) evoluíram para óbito, 161 (57,5\%) para a cura e 12 (4,29\%) foram censurados. O modelo final de sobrevivência foi composto por uso de respirador mecânico para o óbito (RzRiscos =2,86; IC95\%: 1,76-4,64; $p=0,00002)$, sinais hemorrágicos para o óbito (RzRiscos = 2,86; IC95\%: 1,69-4,84; $p=0,00009)$ e para a cura (RzRiscos =0,66; IC95\%: 0,45-0,95; $p=0,03)$, cefaleia para a cura (RzRiscos = 1,99; IC95\%: 1,12-3,54; $p=0,02)$ e menor de 20 anos para a cura (RzRiscos = 1,73; IC95\%: 1,05-2,84; $p=0,03)$. Sinais hemorrágicos e uso de respirador mecânico são fatores relacionados com a gravidade dos casos e não oportunizam tempo suficiente para reverter o desfecho. No entanto, esses sinais de gravidade corroboram o argumento da importância da suspeição oportuna, permitindo um manejo clinico adequado a fim de reduzir a letalidade.

Infecções por Hantavírus; Análise de Sobrevida; Mortalidade
1 Universidade Federal do Paraná, Curitiba, Brasil. 


\section{Introdução}

Hantavirose é uma doença emergente e que tem aumentado a conscientização dos pesquisadores em relação ao seu impacto na saúde pública 1,2,3.

Segundo dados do Ministério da Saúde 4 de 1993 a 2017, o Brasil registrou 2.061 casos de hantavirose e 827 óbitos, com uma letalidade de 40,1\%. A Região Sul apresentou a maior prevalência, com $35,4 \%$ e $29,5 \%$ dos óbitos, com uma letalidade de $33,5 \%$.

Um estudo realizado no Brasil 5 para identificar fatores de risco associados ao óbito nas diferentes regiões do país, encontrou que na Região Sul os indivíduos do sexo masculino tiveram risco de óbito $70 \%$ menor quando comparados com pessoas do sexo feminino.

No Estado de Goiás, a insuficiência respiratória aguda foi o fator que permaneceu associado ao óbito 6. Em Ribeirão Preto (São Paulo), a pneumonia atípica foi associada a um bom prognóstico e a infusão de fluidos superior a $2.000 \mathrm{~mL}$ e a hipotensão arterial associaram-se a um mau prognóstico 7 .

Esses e outros estudos identificando fatores prognósticos ao óbito por hantavirose no processo de revisão bibliográfica demonstraram que a sobrevida em hantavirose é um tema pouco explorado, tendo em vista seu perfil de doença aguda. Entretanto, considerando que seus sinais e sintomas são inespecíficos e facilmente confundidos com outras doenças, seria desejável que seu prognóstico fosse melhorado por meio do conhecimento de fatores que pudessem contribuir para seu desfecho.

O trabalhou objetivou identificar os fatores associados ao tempo de sobrevivência em pessoas com hantavirose no Paraná de 1992 a 2016.

\section{Métodos}

O estudo incluiu casos confirmados de hantavirose no Paraná entre janeiro de 1992 e junho de 2016. A fonte de dados primária foi o Sistema de Informação de Agravos de Notificação (SINAN), cuja inserção e encerramento de fichas epidemiológicas foram acompanhados pelo mesmo técnico no nível estadual em todo o período do estudo. Para os campos sem preenchimento ou inconsistentes foi realizada consulta com a fonte notificadora para melhor completitude do banco. A escolha da data final foi definida em função de ajustes e finalização do banco de dados.

Os casos foram classificados conforme desfecho da infecção por hantavirose: cura ou óbito. Para investigar os efeitos das covariáveis no tempo até um dos desfechos foi ajustado um modelo de análise de sobrevivência com riscos competitivos 8 . Os registros sem informação de data do desfecho foram considerados censuras. A medida de associação foi a razão de riscos (RzRiscos), com os respectivos intervalos de 95\% de confiança (IC95\%).

As covariáveis analisadas foram as demográficas (sexo e idade), os sinais e sintomas (astenia, cefaleia, diarreia, dispneia, dor abdome-toraco-lombar, febre, hipotensão, insuficiência renal, mialgias, náuseas e vômitos, sinais hemorrágicos, sintomas neurológicos, tontura e tosse), os exames complementares (hematócritos, plaquetas, leucócitos alterados, ureia e creatinina aumentados e linfócitos atípicos), resultados de raio $\mathrm{X}$ e suporte terapêutico (respirador mecânico e drogas vasoativas).

As probabilidades de transição dos desfechos foram estimadas utilizando-se o estimador não paramétrico de Aalen-Johansen ${ }^{8}$.

As covariáveis identificadas na univariada com valor de $\mathrm{p}<0,15$ foram consideradas para o modelo múltiplo. Foi utilizado o método stepwise e consideradas significativas as variáveis com valor de $\mathrm{p}<0,05$.

Utilizou-se o EpiInfo, versão 7.2 (https://www.cdc.gov/epiinfo/index.html), e o programa R, versão 3.4.2 (http://www.r-project.org), pacotes Rcmdr, ResourceSelection e mstate.

O estudo foi aprovado no Comitê de Ética em Pesquisa do Setor de Ciências da Saúde da Universidade Federal do Paraná, no dia 11 de novembro de 2016 (parecer no 1.818.206/2016). 


\section{Resultados}

Dos 280 indivíduos com hantavirose, 107 (38,21\%) evoluíram para o óbito, 161 (57,5\%) para a cura e 12 (4,29\%) foram censurados.

Acerca da sobrevida dos casos, observou-se que nos primeiros dias de evolução a probabilidade é maior para o óbito. No entanto, após cerca de uma semana, a probabilidade de cura é maior (Figura 1).

Dentre as covariáveis identificadas associadas ao óbito na univariada foram: ter entre 40 e 49 anos (RzRiscos = 0,48; IC95\%: 0,25-0,91; p = 0,03); dispneia (RzRiscos = 4,2; IC95\%: 2,11-8,37; p = 0,00005); hipotensão (RzRiscos = 7,8; IC95\%: 2,48-24,76; $\mathrm{p}=$ 0,0005); sinais hemorrágicos (RzRiscos = 4,48; IC95\%: 2,79-7,19; p = 0); hematócrito > 46\% (RzRiscos = 2,81; IC95\%: 1,56-5,01; p = 0,0006); leucócitos aumentados com desvio à esquerda (RzRiscos = 1,89; IC95\%: 1,14-3,14; $\mathrm{p}=0$,01); infiltrado pulmonar (RzRiscos = 12,13; IC95\%: 2,97-49,51; p = 0,0005); respirador mecânico (RzRiscos = 3,89; IC95\%: 2,506,05; $\mathrm{p}=$ 0,000000002); e drogas vasoativas (RzRiscos = 3,05; IC95\%: 1,77-5,26; p = 0,00006).

Das covariáveis associadas ao desfecho cura na univariada identificou-se: ter $<20$ anos (RzRiscos = 1,7; IC95\%: 1,07-2,71; p = 0,03); cefaleia (RzRiscos = 1,99; IC95\%: 1,13-3,52; p = 0,02); náuseas e vômitos (RzRiscos = 1,56; IC95\%: 1,08-2,26; p = 0,02); sinais hemorrágicos (RzRiscos = 0,66; IC95\%:

\section{Figura 1}

Gráfico da probabilidade de transição do início dos sintomas até a cura e início dos sintomas até o óbito. Paraná, Brasil, $1992-2016$.

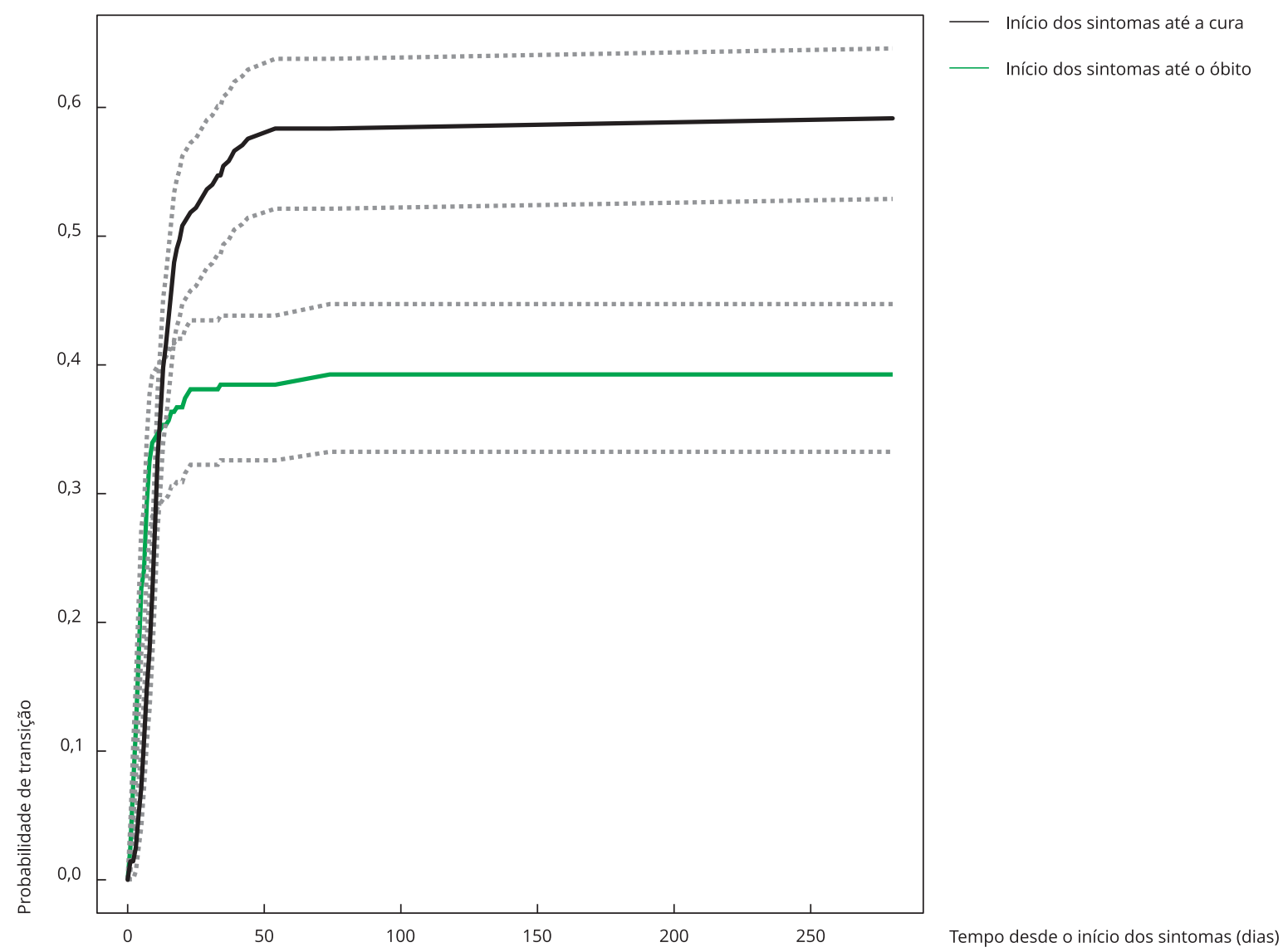

Nota: as linhas pontilhadas são os intervalos de 95\% de confiança para as estimativas das probabilidades de transição. 
0,47-0,92; $\mathrm{p}=0,01)$; ureia e creatinina aumentados (RzRiscos = 0,54; IC95\%: 0,37-0,79; $\mathrm{p}=0,002)$; respirador mecânico (RzRiscos = 0,62; IC95\%: 0,43-0,91; p = 0,01); e drogas vasoativas (RzRiscos = 0,61; IC95\%: 0,38-0,98; $\mathrm{p}=0,04)$.

$\mathrm{Na}$ análise múltipla as covariáveis que permaneceram associadas ao desfecho foram: sexo, $<20$ anos, cefaleia, hipotensão, dispneia, sinais hemorrágicos, ureia e creatinina aumentados, hematócrito $>46 \%$ e respirador mecânico.

O modelo final de sobrevivência, ajustado por sexo e idade e considerando o efeito das covariáveis nos desfechos óbito e cura, foi composto por respirador mecânico para o óbito (RzRiscos = 2,86; IC95\%: 1,76-4,64; $\mathrm{p}=$ 0,00002), sinais hemorrágicos para ambos os desfechos ( $\mathrm{RzRiscos}=2,86$; IC95\%: 1,69-4,84; $\mathrm{p}=0,00009$ e RzRiscos = 0,66; IC95\%: 0,45-0,95; $\mathrm{p}=0$ 0,03, óbito e cura, respectivamente), cefaleia para a cura (RzRiscos = 1,99; IC95\%: 1,12-3,54; p =0,02) e $<20$ anos para a cura (RzRiscos = 1,73; IC95\%: 1,05-2,84; $\mathrm{p}=0,03$ ).

\section{Discussão}

Uma das principais limitações do estudo está no fato de analisar dados cuja coleta e preenchimento foram realizados somente no ato da notificação, não permitindo o conhecimento sobre sua evolução, o que pode ter comprometido a assertividade da análise.

A probabilidade de evolução entre óbitos e curas denotou perfil de doença aguda. Assim, se o indivíduo com hantavirose não vier a óbito na primeira semana, a tendência é de cura. Corroborando com esses achados, no estudo com dados nacionais de 2007 a 2010, o curso da doença até o óbito foi um terço do tempo dos que evoluíram para cura 5 .

A hantavirose se manifesta inicialmente com sintomas como febre, cefaleia, mialgia, náusea e vômito demonstrando o curso benigno da doença. Dos fatores que influenciam a sobrevida, a cefaleia, covariável prognóstica para a cura, pode ser um indicador preditivo importante desde que identificada oportunamente e associada ao histórico epidemiológico ${ }^{9}$ sugestivo para hantavirose. Adicionalmente, melhores chances de sobrevivência dependem da suspeita precoce para o diagnóstico e cuidados intensivos apropriados 10,11.

Outra covariável prognóstica para a cura foi a faixa etária menor de vinte anos, explicada pelo fato dos jovens se recuperarem e tenderem a responder melhor ao tratamento quando comparados aos mais velhos.

Embora a covariável sinais hemorrágicos tenha se apresentado significativa em ambos os desfechos, apresentou risco baixo de cura para casos com sinais hemorrágicos e risco aumentado para o óbito.

Assim, os fatores prognósticos associados ao óbito, como sinais hemorrágicos e uso de respirador mecânico, são fatores já conhecidos e relacionados com a gravidade dos casos. A sua identificação como preditor para o óbito provavelmente não traz oportunidade necessária para reverter o desfecho.

Além disso, a possível associação desses fatores com os tipos de vírus circulantes no sul do país, como os vírus Araucária e Jaborá 12 , cuja apresentação sindrômica é cardiopulmonar, pode ter influenciado na manifestação clínica, letalidade e prognóstico da doença. A fase cardiopulmonar pode progredir rapidamente com descompensação em apenas algumas horas 13 .

Embora o método adotado neste estudo tenha sido diferente dos trabalhos que buscam identificar fatores prognósticos, os achados encontrados corroboram com a literatura 5,14,15,16,17.

Os resultados deste estudo evidenciaram a importância da suspeição oportuna, permitindo um manejo clínico adequado a fim de reduzir as taxas de letalidade 14,15. 


\section{Colaboradores}

D. A. Arita e S. E. Shimakura contribuíram com a concepção do projeto, análise e interpretação dos dados, redação do artigo, revisão crítica relevante do conteúdo intelectual e aprovação final da versão a ser publicada; são responsáveis por todos os aspectos do trabalho na garantia da exatidão e integridade da obra.

\section{Referências}

1. Badra SJ, Maia FG, Figueiredo GG, Santos Junior GS, Campos GM, Figueiredo LT, et al. A retrospective serologic survey of hantavírus infectious in the county of Cassia dos Coqueiros. Rev Soc Bras Med Trop 2012; 45:46870.

2. Raboni SM, Rubio G, Borba L, Zeferino A, Skraba I, Goldenberg S, et al. Clinical survey of hantavirus in Southern Brazil and the development of specific molecular diagnosis tools. Am J Trop Med Hyg 2005; 72:800-4.

3. Oliveira SV, Fonseca LX, Barros e Silva PMR, Pereira SVC, Caldas EP. Análise do perfil epidemiológico da hantavirose no Brasil no período de 2007 a 2012. Rev Patol Trop 2014; 43:131-42.

4. Ministério da Saúde. Situação epidemiológica - dados hantavirose. http://portalms.saude. gov.br/saude-de-a-z/hantavirose/11304-situ acao-epidemiologica-dados (acessado em 28/ Mai/2018).

5. Willemann MCA, Oliveira SV. Risk factors associated with hantavirosis fatality: a regional analysis from a case-control study in Brazil. Rev Soc Bras Med Trop 2014; 47:47-51.

6. Menezes Filho HR, Moreli ML, Souza ALL, Costa VG. Estudo transversal da letalidade da hantavirose no Estado de Goiás, 2007-2013. Epidemiol Serv Saúde 2016; 25:519-30.

7. Campos GM, Borges AA, Badra SJ, Figueiredo GG, Souza RLM, Moreli LM, et al. Síndrome pulmonar e cardiovascular por hantavírus: aspectos clínicos de uma doença emergente no sudeste brasileiro. Rev Soc Bras Med Trop 2009; 42:282-9.

8. Geskus RB. Data analysis with competing risks and intermediate states. New York: Chapman \& Hall; 2016.

\section{Informações adicionais}

ORCID: Daniele Akemi Arita (0000-0003-11029063); Silvia Emiko Shimakura (0000-0002-54682516).

9. Santos VM, Rocha de Sá DA, Turra TZ, Ferreira Borges NM, Nascimento UM, Damasceno EA. Hantavirus pulmonary syndrome in Brasilia periphery: a diagnostic challenge. J Infect Dev Ctries 2009; 3:639-43.

10. Bi Z, Formenty PBH, Roth CE. Hantavirus infection: a review and global update. J Infect Dev Ctries 2008; 2:3-23.

11. Stollenwerk N, Harper RW, Sandrock CE. Bench-to-bedside review: rare and common viral infections in the intensive care unit linking pathophysiology to clinical presentation. Crit Care 2008; 12:219.

12. Raboni SM, Delfraro A, de Borba L, Teixeira BR, Stella V, de Araujo MR, et al. Hantavirus infection prevalence in wild rodents and human anti-hantavirus serological profiles from different geographic areas of South Brazil. Am J Trop Med Hyg 2012; 87:371-8.

13. Hartline J, Mierek C, Knutson T, Kang C. Hantavirus infection in North America: a clinical review. Am J Emerg Med 2013; 31:978-82.

14. Kaya S. Prognostic factors in hantavirus infections. Mikrobiyol Bul 2014; 48:179-87.

15. Riquelme R, Rioseco ML, Bastidas L, Trincado D, Riquelme M, Loyola $\mathrm{H}$, et al. Hantavirus pulmonary syndrome, Southern Chile, 19952012. Emerg Infect Dis 2015; 21:562-8.

16. Elkhoury MR, Mendes WS, Waldman EA, Dias JP, Carmo EH, Vasconcelos PFC. Hantavirus pulmonary syndrome: prognostic factors for death in reported cases in Brazil. Am J Trop Med Hyg 2012; 106:298-302.

17. Oliveira RC, Sant'Ana MM, Guterres A, Fernandes J, Hillesheim NLFK, Lucini C, et al. Hantavirus pulmonary syndrome in a highly endemic área of Brazil. Epidemiol Infect 2015; 144:1096-106. 


\section{Abstract}

Hantavirus infection is an emerging disease with public health impact. Since the signs and symptoms are nonspecific and easily confused with those of other diseases, the prognosis could be improved by knowledge of the factors potentially contributing to the outcome. The study aimed to identify the factors associated with survival time in persons with hantavirus infection. This was a study of confirmed cases of hantavirus infection from January 1992 to June 2016 in Paraná State, Brazil. Each case presented two possible competitive outcomes: death or cure. Survival analysis used a competitive risks model and outcome probabilities estimated by the Aalen-Johansen estimator. The measure of association was the hazards ratio (HR) with $95 \%$ confidence interval $(95 \% \mathrm{CI})$. Of the 280 individuals with hantavirus infection, 107 (38.21\%) evolved to death, 161 (57.5\%) to cure, and $12(4.29 \%)$ were censored. The final survival model consisted of the use of mechanical ventilation for death $(H R=2.86$; 95\% CI: 1.76-4.64; $p=0.00002)$, hemorrhagic signs for death $(H R=2.86 ; 95 \% C I: 1.69-4.84 ; p=0.00009)$ and for cure (HR $=0.66 ; 95 \% C I: 0.45-0.95 ; p=0.03)$, headache for cure (HR = 1.99; 95\% CI: 1.12-3.54; $p=0.02)$, and age under 20years forcure $(H R=1.73$; 95\%CI: 1.05-2.84; $p=0.03$ ). Hemorrhagic signs and use of mechanical ventilation are factors associated with the severity of cases and do not allow sufficient time to reverse the outcome. However, these signs of severity corroborate the argument of the importance of timely suspicion, allowing adequate clinical management to reduce case-fatality.

Hantavirus Infections; Survival Analysis; Mortality

\section{Resumen}

El hantavirus es una enfermedad emergente y con impacto en la salud pública. Por presentar señales $y$ sintomas inespecíficos, $y$ fácilmente confundidos con otras enfermedades, su pronóstico podría ser mejor gracias al conocimiento de factores que pudiesen contribuir a su resultado. El estudio tuvo como objetivo identificar los factores asociados al tiempo de supervivencia en personas con hantavirus. Se trata de un estudio con casos confirmados de hantavirus de enero/1992 a junio/2016 del estado de Paraná, Brasil. Cada caso presentó dos desenlaces competitivos posibles: óbito o cura. Para el análisis de supervivencia se utilizó un modelo de riesgos competitivos y las probabilidades de los resultados se estimaron mediante el estimador de Aalen-Johansen. La medida de asociación fue la razón de riesgos (RzRiscos), con sus respectivos intervalos de 95\% de confianza (IC95\%). De los 280 individuos con hantavirus, 107 (38,21\%) evolucionaron hacia el óbito, $161(57,5 \%)$ hacia la cura y $12(4,29 \%)$ fueron censurados. El modelo final de supervivencia estuvo compuesto por el uso de respirador mecánico para el óbito (RzRiscos $=2,86$; IC95\%: 1,76-4,64; $p=0,00002)$, señales hemorrágicas para el óbito (RzRiscos = 2,86; IC95\%: 1,69-4,84; $p=0,00009)$ y para la cura $($ RzRiscos $=$ 0,66; IC95\%: 0,45-0,95; $p=0,03)$, cefalea para la cura (RzRiscos = 1,99; IC95\%: 1,12-3,54; $p=0,02)$ y menor de 20 años para la cura (RzRiscos = 1,73; IC95\%: 1,05-2,84; $p=0,03)$. Las señales hemorrágicas y uso de respirador mecánico son factores relacionados con la gravedad de los casos y no destinar tiempo suficiente para revertir el desenlace. No obstante, estas señales de gravedad corroboran el argumento de la importancia de la sospecha oportuna, permitiendo un manejo clínico adecuado, con el fin de reducir la letalidad.

Infecciones por Hantavirus; Análisis de Supervivencia; Mortalidad
Recebido em 30/Mai/2018

Versão final reapresentada em 19/Jan/2019

Aprovado em 28/Jan/2019 